\title{
Probabilistic Approach to the Service Life Prediction of Timber Claddings
}

\author{
Ana Silva ${ }^{1}$ and Andrés José Prieto ${ }^{2}$ \\ ${ }^{1}$ Department of Civil Engineering, Architecture and Georresources, Instituto Superior Técnico, \\ University of Lisbon, Lisbon, Portugal, ana.ferreira.silva@tecnico.ulisboa.pt \\ ${ }^{2}$ Instituto de Arquitectura y Urbanismo, Universidad Austral de Chile, Edificio Ernst Kasper (Campus \\ Isla Teja),Valdivia, Chile, andres.prieto@uach.cl
}

\begin{abstract}
The service life of buildings and components is extremely difficult to model and predict, mainly due to the uncertainty and variability associated with the degradation phenomena. These uncertainties are usually related with the intrinsic randomness of natural phenomena, such as the environmental degradation agents. In order to deal with these uncertainties, in this study, a stochastic approach is used, to model the service life of timber claddings. For this purpose, a logistic regression analysis is used to evaluate the probability of timber claddings to reach the end of their service life over time. Moreover, the impact of the environmental exposure conditions (e.g. facades orientation, distance from the ocean, exposure to damp and exposure to wind/rain action), which influence the degradation and service life of timber claddings, is also evaluated. The proposed models are applied to 150 timber claddings, located in Valdivia (South Chile), which have been analysed during an extensive fieldwork survey. The results obtained in this study aid the definition of maintenance plans, in a technically informed manner, in order to promote the conservation of these claddings, which are an important symbol of the local identity of this region, depicting a complexity of cultural values, inherited by Spanish and German colonisations.
\end{abstract}

Keywords: Durability, Service Life Prediction, Timber Claddings, Chile.

\section{Introduction}

A rational approach to the definition of maintenance policies and strategies requires an efficient evaluation of the service life of the building or element under analysis. External claddings are one of the most vulnerable elements of a construction, since are subjected to several degradation agents and mechanisms (Silva et al., 2016). The façade is considered as a building component, acting as the first durability layer, contributing to the building's performance and aesthetic appearance, functioning as the interface of the exterior and the inner environment, thus also contributing to the users' internal comfort. The deterioration rate of timber façades depends on several variables, including location, solar orientation, architectonical details, exposure level, microclimate, and intrinsic properties of materials used in construction. The use of service life prediction methodologies allows a more rational management of the maintenance of buildings and its constructed components by supporting reduced life cycle costs of built assets. This study evaluates the probability distribution of the degradation condition of timber claddings, using the evaluation of the characteristics of 150 cladding systems inspected in Valdivia, Chile. For that purpose, a logistic regression analysis is used, which allows forecasting the probability of the timber claddings to reach the end of their service life over time and according to their environmental exposure conditions. This analysis is relevant in developing countries where there are few studies related to the service life prediction of building components (façades). The scarcity of funds to maintain and preserve the built park and infrastructures needs a more rational approach to decision-making regarding the inspection, maintenance and rehabilitation. 


\subsection{Degradation Phenomena of Timber Claddings}

In 2011, Gaspar and de Brito (2011) proposed a numerical index (severity of degradation - $S_{w}$ ) to express the overall degradation condition of facades claddings. The severity of degradation $\left(S_{w}\right)$ index is obtained by the ratio between the weighted degraded area and the total cladding area, assuming the highest possible level of degradation (equation 1). This methodology was originally developed for rendered facades, establishing a general framework model, and was adapted to new cladding materials, as natural stone claddings, ceramic cladding systems, painted surfaces (Silva et al., 2016).

$$
S_{w}=\frac{\sum\left(A_{n} \times k_{n} \times k_{a, n}\right)}{A \times \sum k_{\max }}
$$

Where $S_{w}$ represents the severity of degradation of timber claddings, expressed as a percentage, $k_{n}$ is the multiplying factor of anomaly " $n$ ", as a function of their degradation level, within the range $\mathrm{K}=\{0,1,2,3,4\}, k_{a, n}$ is the weighting factor corresponding to the relative weight of the anomaly detected, $A_{n}$ is the area of cladding affected by an anomaly " $n$ ", in $m^{2}, A$ is the facade area, in $\mathrm{m}^{2}$, and $\sum\left(k_{\max }\right.$. $)$ is the sum of the weighting factors for the highest degradation level of each anomaly type in a cladding with an area equal to $A$. In Table 1 , the different types of anomalies detected are classified according to five degradation levels, considering the extent of the cladding affected by them. The degradation levels proposed vary between level A (very good condition) - the most favourable condition, corresponding to claddings with no visible degradation - and level E (severe degradation) - the worst possible

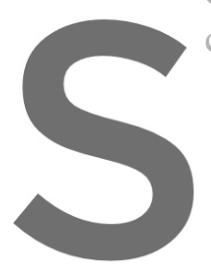

condition, corresponding th
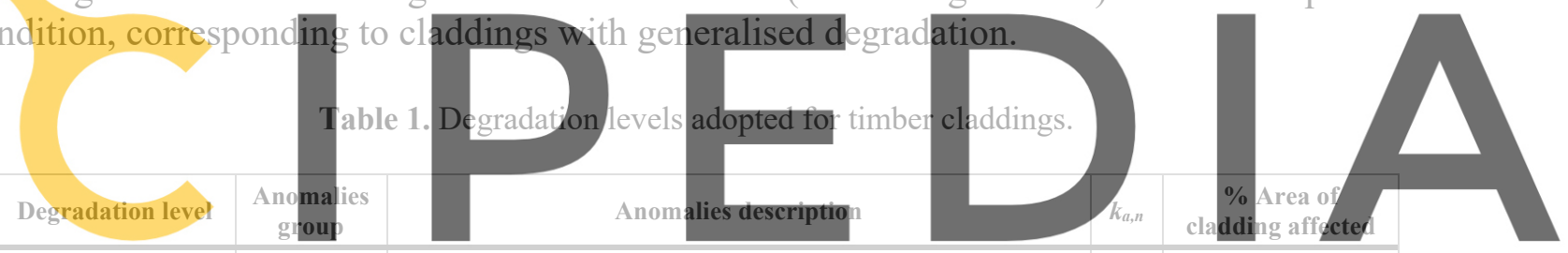

Level A

Register for free at $\begin{gathered}\text { (Very good) } \\ \text { No visible degradation }\end{gathered}$

\begin{tabular}{|c|c|c|c|c|}
\hline \multirow{3}{*}{$\begin{array}{c}\text { Level B } \\
(\text { Good condition) } \\
\mathbf{1 \%}<\boldsymbol{S}_{w} \leq \mathbf{1 0 \%}\end{array}$} & \multirow{2}{*}{ Aesthetic } & AA1 Staining or color change (dirty surface) & 0.3 & $\leq 15 \%$ \\
\hline & & AA2 Wearing or detachment of the finishing layer & 0.7 & $\leq 10 \%$ \\
\hline & $\begin{array}{l}\text { Loss of } \\
\text { integrity }\end{array}$ & AB1 Warping, swelling or other flatness deficiencies & 0.5 & $\leq 10 \%$ \\
\hline \multirow{4}{*}{$\begin{array}{c}\text { Level C } \\
\text { (Slight } \\
\text { degradation) }\end{array}$} & \multirow{2}{*}{ Aesthetic } & AA1 Staining or color change (dirty surface) & 0.3 & $>15 \%$ \\
\hline & & AA2 Wearing or detachment of the finishing layer & 0.7 & $>10 \%$ to $\leq 50 \%$ \\
\hline & \multirow{5}{*}{$\begin{array}{c}\text { Loss of } \\
\text { integrity }\end{array}$} & AB1 Warping, swelling or other flatness deficiencies & 0.5 & $>10 \%$ to $\leq 50 \%$ \\
\hline & & $\begin{array}{c}\text { AB2 Cracking of elements and/or joints (possibly from substrate } \\
\text { cracking) }\end{array}$ & 0.9 & $\leq 20 \%$ \\
\hline \multirow{3}{*}{$10 \%<S_{w} \leq 20 \%$} & & AB6 Moisture (Oxyde) / Biocostra & 0.5 & $\leq 30 \%$ \\
\hline & & AB8 Delamination & 1.0 & $\leq 10 \%$ \\
\hline & & AB9 Crumbling & 1.2 & $\leq 10 \%$ \\
\hline \multirow{10}{*}{$\begin{array}{c}\text { Level D } \\
\text { (Moderate } \\
\text { degradation) }\end{array}$} & Aesthetic & AA2 Wearing or detachment of the finishing layer & 0.7 & $>50 \%$ \\
\hline & \multirow{9}{*}{$\begin{array}{c}\text { Loss of } \\
\text { integrity }\end{array}$} & AB1 Warping, swelling or other flatness deficiencies & 0.5 & $>50 \%$ \\
\hline & & $\begin{array}{c}\text { AB2 Cracking of elements and/or joints (possibly from substrate } \\
\text { cracking) }\end{array}$ & 0.9 & $>20 \%$ \\
\hline & & AB3 Broken or splintered elements & 1.0 & $\leq 20 \%$ \\
\hline & & AB4 Detachment of elements from substrate (lack of adherence) & 1.2 & $\leq 10 \%$ \\
\hline & & AB6 Moisture (Oxyde) / Biocostra & 0.5 & $>30 \%$ \\
\hline & & AB5 Rot & 1.2 & $\leq 10 \%$ \\
\hline & & AB7 Xylophage attack & 1.0 & $\leq 10 \%$ \\
\hline & & AB8 Delamination & 1.0 & $>10 \%$ to $\leq 30 \%$ \\
\hline & & AB9 Crumbling & 1.2 & $>10 \%$ to $\leq 30 \%$ \\
\hline \multirow{6}{*}{$\begin{array}{c}\text { Level E } \\
\text { (Severe } \\
\text { degradation) }\end{array}$} & \multirow{6}{*}{$\begin{array}{c}\text { Loss of } \\
\text { integrity }\end{array}$} & AB3 Broken or splintered elements & 1.0 & $>20 \%$ \\
\hline & & AB4 Detachment of elements from substrate (lack of adherence) & 1.2 & $>10 \%$ \\
\hline & & AB5 Rot & 1.2 & $>10 \%$ \\
\hline & & AB7 Xylophage attack & 1.0 & $>10 \%$ \\
\hline & & AB8 Delamination & 1.0 & $>30 \%$ \\
\hline & & AB9 Crumbling & 1.2 & $>30 \%$ \\
\hline
\end{tabular}




\section{Probabilistic Analysis of Timber Claddings Reaching the End of their Service Life Over Time}

A logistic regression analysis is used to model the probability of timber claddings reaching the end of their service life over time and according to their environmental exposure conditions. Logistic regression is one of the most used statistical procedures, which allows describing the relationship between a categorical dependent variable and one or more explanatory variables, in terms of the probability of a given event. In this study, the dependent variable corresponds to the question "Has the timber cladding reached the end of its service life?", and this question presents two mutually exclusive classes: "yes" and "no". For the timber claddings under analysis, it is assumed that a cladding reaches the end of its service life, when presents a set of anomalies corresponding to a severity of degradation of $20 \%$. Figure 1 shows an illustrative example of a timber cladding in the end of its service life. The limit to establish the end of service life is conventionally defined and is related to the Chilean context, and with the users' acceptance criteria. This theoretical limit can change according to the buildings' economic and social context or even funds available for maintenance actions. The sample analysed is composed by 150 timber claddings, located in Valdivia (South Chile), and only 8\% of the claddings have reached the end of their service life. In the first analysis performed, only the age of the claddings is used as explanatory variable.
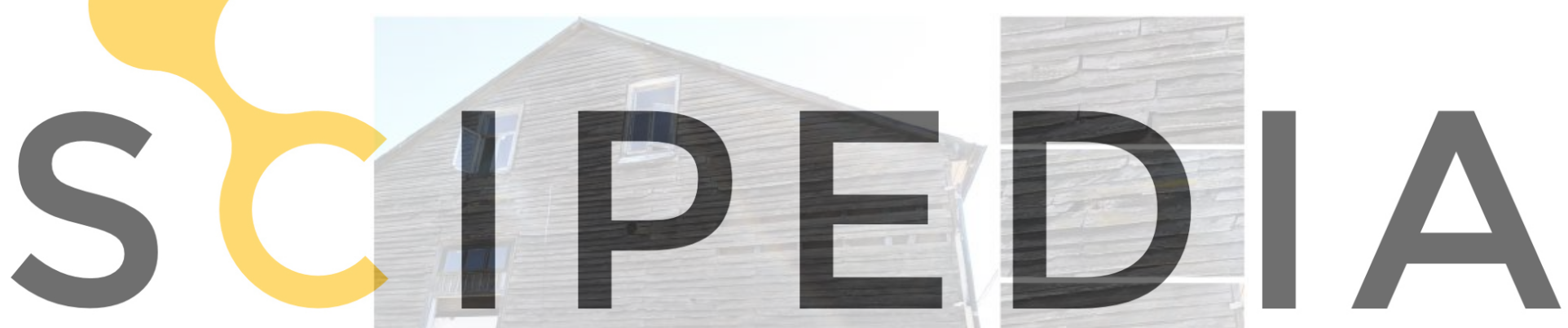

Register for free at https//www.scipedia.com to download the version without the watermark Figure 1. Case study that exemplifies the end of the service life of timber claddings.

The proposed model, described by Equation (2), allows evaluating the probability of a timber cladding reach the end of its service life according to its age. The goodness-of-fit of the proposed models to the dataset under analysis is evaluated through the Chi-square test $\left(\chi^{2}\right)$ and the Chi-square test of Hosmer-Lemeshow $\left(\chi_{\mathrm{HL}}{ }^{2}\right)$. These tests are easy to compute and are present in the statistical software used in this study; furthermore, as mentioned in Silva et al. (2016), these tests have been widely used mainly due to the nonexistence of better approaches. For the Chi-square test $\left(\chi^{2}\right)$ test, a $p$-value around 1 reveals a better fit to data than a model that only has the constant; on the other hand, a $p$-value around 1 for $\chi_{\mathrm{HL}^{2}}$ test, reveals that the age of the claddings or the other independent variables included in the model, are relevant for the end of service life of timber claddings. The model summary $\left(\chi^{2}(148)=17.826, p=1.00\right)$ and the Hosmer and Lemeshow test $\left(\chi_{\mathrm{HL}}^{2}(8)=0.133, p=1.00\right)$ reveal an adequate adjustment of the model to the sample under analysis. The Wald test $\left(\chi\right.$ wald $^{2}(1)=12.163$, with $\left.p=0.00\right)$ shows that the age is statistically relevant to predict the probability of timber claddings reaching the end of their service life.

$$
P(Y=\text { "End of service life" })=1-\frac{1}{1+e^{-18.200+0.539 A g e}}
$$


Figure 2 shows the probabilistic distribution of timber claddings reaching the end of their service life over time. As expected, the probability of timber claddings reaching the end of their service life increases with the claddings' age. After 33.8 years, a timber cladding presents a probability higher than $50 \%$ of have reached the end of its service life, after 35.8 years, this probability is higher than $75 \%$ and after 37.9 years, it is higher than $90 \%$.

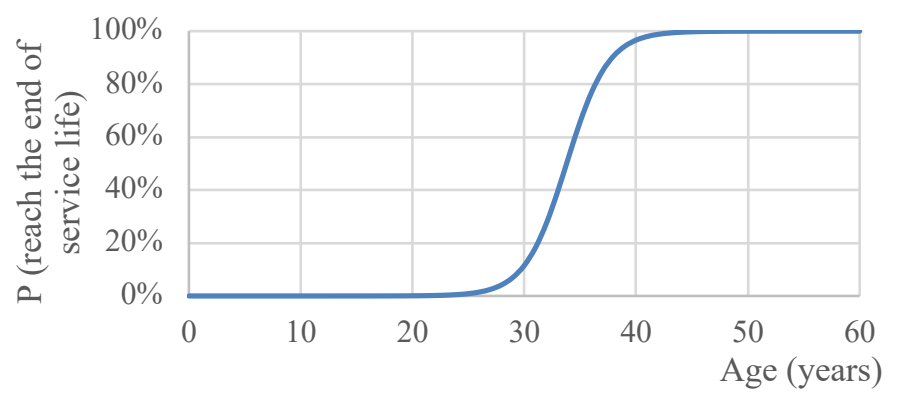

Figure 2. Distribution of probability of timber claddings reaching the end of their service life over time.

Table 2 shows the classification table of the proposed model, which allows evaluating the percentage of timber claddings correctly classified by the model, i.e. the classification table provides the accuracy of the model, evaluating the capability of the model discriminate the case studies that has reached the end of their service life from the case studies that has not reached this limit. The proposed model classifies correctly $97.3 \%$ of the timber claddings analysed, revealing that the model presents a good classification capability.

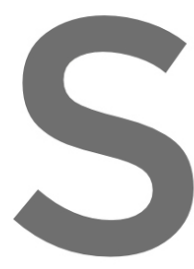

Register for free at the timber cladding reached. No

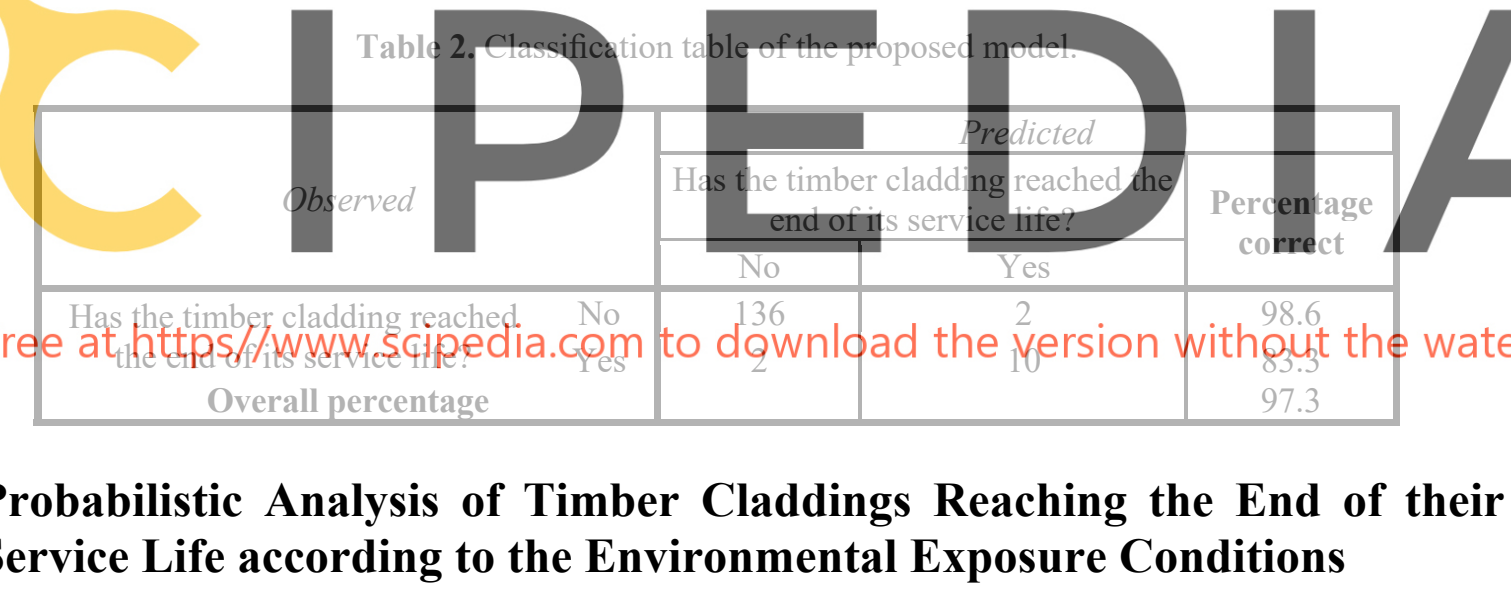

Wood is frequently used as external cladding, mainly due to aesthetic, ecological or architectural reasons. Nevertheless, wood is extremely susceptible to various degradation agents and mechanisms (e.g. wetting, microorganisms, fungi or algae, xylophage agents), especially related with the environmental exposure conditions (Brischke et al., 2017). In this sense, in this study, the influence of the environmental exposure conditions in the service life of timber claddings, located in Valdivia (Chile) is analysed. For this purpose, the influence of the exposure to damp, the façades orientation, the wind-rain action and the exposure to sea salts (distance from Pacific Ocean) is evaluated. In Figure 3, the probability of timber claddings reaching the end of their service life over time, according to their exposure to damp, is analysed. For the exposure to damp, two categories are considered (Silva et al., 2016): i) high, for claddings located near to damp sources, in contact with the terrain, in areas with high relative humidity, and not protected by adjacent buildings; and ii) medium, for claddings that are not directly exposed to damp sources. Equation (3) allows estimating the probability of a timber cladding to reach the end of its service life over time and according to its exposure to damp, where the variable "Age" must be replaced by the age of the timber claddings and the variable "Damp" must be replaced by 1 if the timber cladding presents a high exposure to damp and by 
0 if the cladding shows a medium exposure to damp. This model correctly classifies $97.3 \%$ of the timber claddings under analysis.

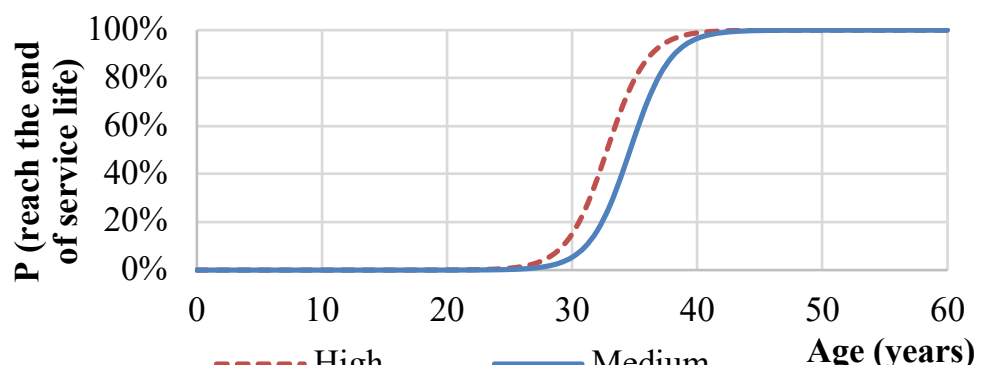

Figure 3. Distribution of the probability of timber claddings reaching the end of their service life over time, according to their exposure to damp.

$$
P(Y=\text { "End of service life" })=1-\frac{1}{1+e^{-21.462+0.620 \text { Age+1.143Damp }}}
$$

Figure 4 shows the probability of timber claddings reaching the end of their service life over time, according to façades orientation. This probability can be estimated using Equation (4), where in the variable "façades orientation", the symbol of the category corresponding to the timber cladding analysed should be replaced by 1 , and the remaining categories' symbols by 0 . The logistic regression model obtained, adopting the variable age and façades orientation correctly classifies $98 \%$ of the sample analysed.
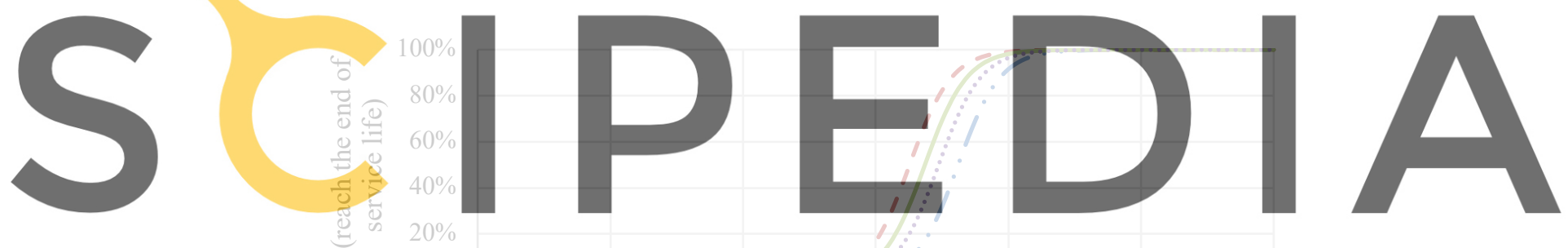

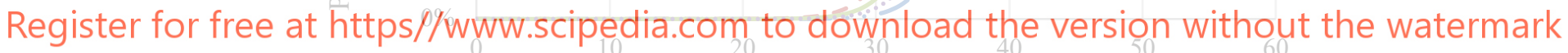

Figure 4. Distribution of the probability of timber claddings reaching the end of their service life over time, according to façades orientation.

$$
P(Y=\text { "End of service life" })=1-\frac{1}{1+e^{-21.826+0.628 A g e-0.921 E+1.375 N / N E / N W+0.652 S / S E / S W}}
$$

Figure 5 presents the probability of timber claddings reaching the end of their service life over time, according to their exposure to wind-rain action. In this study, two categories are adopted to characterise the exposure conditions of timber claddings to the combined action of wind and rain: i) unfavourable, for claddings located in tall buildings, located in areas unsheltered by surrounding buildings or vegetation, thus exposed to prevailing winds; and ii) current, for claddings protected from the direct incidence of rain and prevailing winds. Equation (5) allows evaluating the probability of a timber cladding to reach the end of its service life according to its age and exposure to wind-rain action, where the variable "Wind" must be replaced by 1 if the timber cladding presents an unfavourable exposure to wind-rain action and by 0 if the cladding shows a current exposure. This model correctly classifies $97.3 \%$ of the timber claddings analysed. 


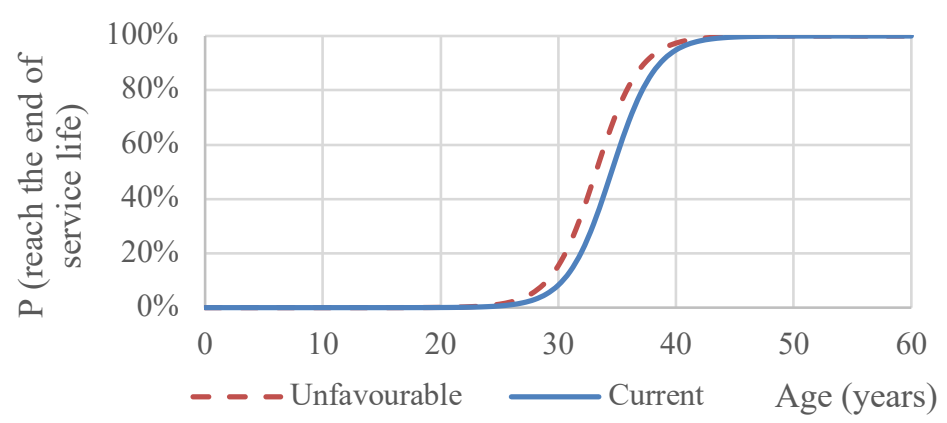

Figure 5. Distribution of the probability of timber claddings reaching the end of their service life over time, according to their exposure to wind-rain action.

$$
P(Y=\text { "End of service life" })=1-\frac{1}{1+e^{-18.369+0.532 \text { Age }+0.703 \text { Wind }}}
$$

In Figure 6, the probability of timber claddings reaching the end of their service life over time, according to their distance from the Pacific Ocean, is shown. In this analysis, two categories are considered: i) claddings located at more than $5 \mathrm{~km}$ from the Pacific Ocean; and ii) claddings located at less than $5 \mathrm{~km}$ from the Pacific Ocean. Equation (6) allows estimating this probability, where the variable "Ocean" must be replaced by 1 if the cladding is located at less than $5 \mathrm{~km}$ from the Pacific Ocean and by 0 if the cladding is located at more than $5 \mathrm{~km}$. This model presents a classification capability of $97.3 \%$.
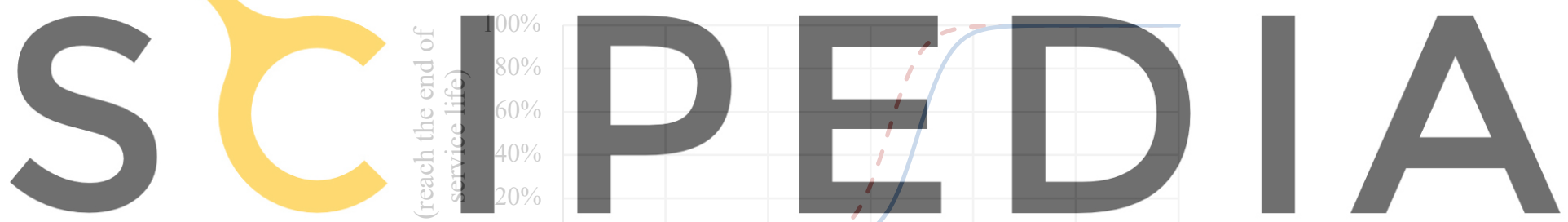

Register for free at https//www.scipedia.com to download the version ${ }^{60}$ without the watermark

Figure 6. Distribution of the probability of timber claddings reaching the end of their service life over time, according to their distance from the Pacific Ocean.

\section{Results and Discussion}

Bio-materials, as timber, when applied in façade claddings, are exposed to several degradation agents, which generally are biotic agents (fungi, insects, moulds, algae) and abiotic agents (water, solar radiation, gradient of temperature, pollutants) (Sandak et al., 2019). Other factors, such as the building orientation and its surrounding environment, presence of adjacent buildings, vegetation, and the proximity of water reservoirs (Silva et al., 2016; Prieto and Silva, 2019), can also compromise the durability of timber claddings. Moreover, the degradation mechanisms usually occur simultaneously, affecting the degradation pattern over time and the extent damages among the different timber claddings analysed. Based on the sample analysed (150 timber claddings located in South Chile), and according to the stochastic models proposed, the following conclusions can be drawn (Table 3).

This analysis reveals that after 33.8 years, a timber cladding presents a probability higher than $50 \%$ of have reached the end of its service life and after 37.9 years, this probability is higher than 90\%. These results are in accordance with the literature related with the durability of timber claddings, which suggests an estimated service life between 30 to 35 years for wood materials in outdoor exposure environments. 
The weathering of timber claddings occurs mainly due to abiotic agents, which present a significant impact in the degradation process of timber claddings in South Chile due to their exposition to severe climatic conditions. In most cases, weathering leads to cracking, colour changes or alterations in the finishing layer (Sandak et al., 2019).

A high exposure to damp contributes to the presence of fungi decay, insects, moulds and algae growth, among other biotic factors (Sandak et al., 2019). Although the presence of mould does not affect the structural properties of wooden façades, it has a large effect on the aesthetical service life of the cladding. Mould leads to the claddings' discolouration, unpleasant odour, and health problems. This factor usually appears in environmental conditions like in Valdivia, where relative humidity $(\mathrm{RH})$ is higher than $75 \%$ and the temperature ranges between 0 and 45 ${ }^{\circ} \mathrm{C}$. Algae, which promotes the colour change of timber claddings, normally occurs in contexts with $\mathrm{RH}>95 \%$ and temperature fluctuations from $0^{\circ} \mathrm{C}$ to $45^{\circ} \mathrm{C}$. Timber claddings with high exposure to damp reach the end of their service life sooner (with a probability of $95 \%$ of have reached the end of its service life after 37.5 years - Table 3 ), than claddings with a low exposure, which reach the end of their service life around two years later (39.4 years). In the sample analysed, timber claddings with high exposure to damp have a higher incidence of rot and xylophage attack.

Regarding the surfaces' orientation, claddings facing North are more exposed to UV radiation, suffering significant thermal gradients. On the other hand, timber claddings facing South, in Chile, are more exposed to the presence of damp. Claddings facing East and West are the most affected by wind-rain action in Valdivia. Table 3 shows that timber claddings facing North reach the end of their service life sooner (with a probability higher than $95 \%$ of reaching

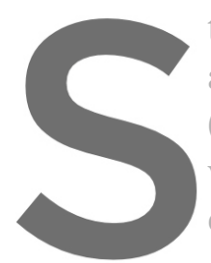
the end of their servide and by the claddings facir (Table 3). The main ano wearing or detachment of the elements from substrate
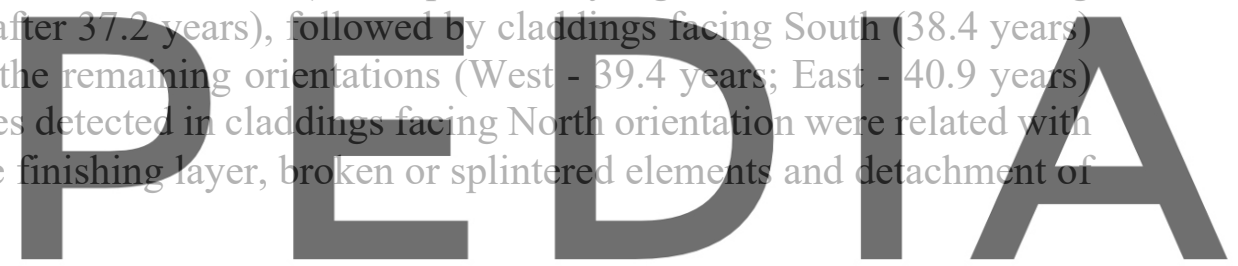

Table 3. Analysis of the probability of timber claddings reach the end of their service life, according to their

Register for free at https//WWW.scipedila.com to opwwhload the

\begin{tabular}{|c|c|c|c|c|c|}
\hline \multicolumn{2}{|c|}{$\begin{array}{l}\text { Environmental exposure } \\
\text { conditions }\end{array}$} & $\begin{array}{l}\text { timber cladding } \\
\text { achieves a } \\
\text { probability of } \\
50 \% \text { of have } \\
\text { reached the end } \\
\text { of its service life }\end{array}$ & $\begin{array}{l}\text { timber cladding } \\
\text { achieves a } \\
\text { probability of } \\
75 \% \text { of have } \\
\text { reached the end } \\
\text { of its service life }\end{array}$ & $\begin{array}{l}\text { timber cladding } \\
\text { achieves a } \\
\text { probability of } \\
90 \% \text { of have } \\
\text { reached the end of } \\
\text { its service life }\end{array}$ & $\begin{array}{l}\text { timber cladding } \\
\text { achieves a } \\
\text { probability of } \\
95 \% \text { of have } \\
\text { reached the end } \\
\text { of its service life }\end{array}$ \\
\hline \multirow{2}{*}{$\begin{array}{l}\text { Exposure to } \\
\text { damp }\end{array}$} & High & 32.8 & 34.6 & 36.4 & 37.5 \\
\hline & Medium & 34.7 & 36.4 & 38.2 & 39.4 \\
\hline \multirow{4}{*}{$\begin{array}{l}\text { Facades } \\
\text { orientation }\end{array}$} & $\mathrm{N}$ & 32.6 & 34.3 & 36.1 & 37.2 \\
\hline & $\mathrm{S}$ & 33.7 & 35.5 & 37.2 & 38.4 \\
\hline & $\mathrm{W}$ & 34.4 & 36.5 & 38.3 & 39.4 \\
\hline & $\mathrm{E}$ & 36.2 & 38.0 & 39.7 & 40.9 \\
\hline \multirow{2}{*}{$\begin{array}{l}\text { Exposure to wind } \\
\text { and rain action }\end{array}$} & Unfavourable & 33.2 & 35.3 & 37.4 & 38.8 \\
\hline & Current & 34.5 & 36.6 & 38.7 & 40.1 \\
\hline \multirow{2}{*}{$\begin{array}{c}\text { Distance from the } \\
\text { Pacific Ocean }\end{array}$} & $<5 \mathrm{~km}$ & 31.7 & 33.5 & 35.5 & 36.5 \\
\hline & $>5 \mathrm{~km}$ & 34.6 & 36.4 & 38.2 & 39.3 \\
\hline
\end{tabular}

The level of exposure to the combined action of wind and rain is influenced by the façades orientation, as well as by the height of the building, the presence of adjacent buildings or even vegetation. In this study, façades with an unfavourable exposure to wind-rain action reach the end of the service life sooner (with a probability higher than $95 \%$ of reaching the end of their service life, after 38.8 years), when compared with façades with a current exposure (40.1 years). 
In the sample analysed, timber claddings with a high exposure to wind and rain are more prone to present staining or colour change, cracking and presence of crumbling.

Finally, timber surfaces close to the Pacific Ocean $(<5 \mathrm{~km})$ reached the end of their service life sooner (with a probability higher than $95 \%$ of reaching the end of their service life, after 36.5 years) than cases located at more than $5 \mathrm{~km}$ from the Ocean coastline ( 39.3 years) (Table 3). Timber claddings closer to the seashore show a higher incidence of anomalies such as cracking of elements and/or joints and wearing or detachment of the finishing layer.

\section{Conclusions}

In this study, a logistic regression analysis is used to evaluate the probability of timber claddings to reach the end of their service life over time and according to the claddings' environmental exposure conditions (e.g. facades orientation, distance from the ocean, exposure to damp and exposure to wind/rain action). According to the proposed model, the 150 timber claddings analysed present a probability higher than $50 \%$ of reaching the end of their service life after 34 years, which is in accordance with the literature and the empirical knowledge related with the degradation of these claddings. As expected, timber façades with unfavourable conditions (closer to the Pacific Ocean, facing North (N) and South (S) orientations and with high exposure to environmental agents, i.e. damp and wind-rain actions) are more prone to reach sooner the end of their service life. In the sample analysed, the most common anomalies detected were staining or colour change; wearing or detachment of the finishing layer; cracking of elements and/or joint; warping, swelling or other flatness deficiencies; and broken or splintered elements.

This model allows predicting the end of service life of timber claddings, i.e. the instant after which is necessary to intervene, jn order to imp
cladding is able to fulfil the users and oyners'
In this sense, the model allows knowing the risk
by the model, and the risk of failure of the ti
environmental degradation agents that influend
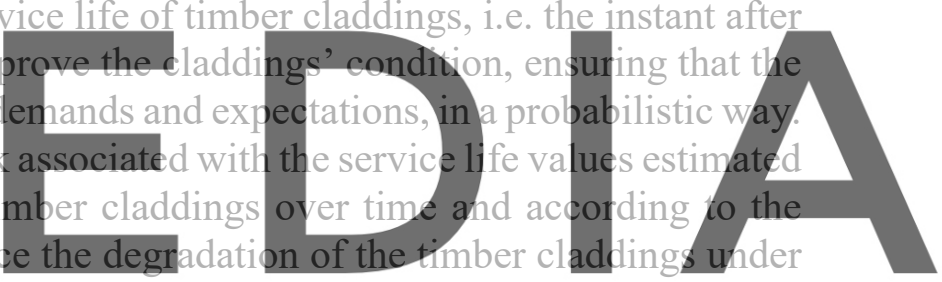

analysis. The results obtained can be useful for a further knowledge of the durability and service

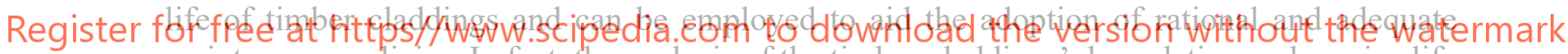
maintenance policies. In fact, the analysis of the timber claddings' degradation and service life in South Chile (Los Rios region), allows establishing new and adapted strategies and maintenance plans, to reduce the repair costs and to optimise the use of scarce resources, as well as promoting new approaches to maintain cultural identities of the southern regions of Chile, in which the wood is one of the main natural resources.

\section{ORCID}

Ana Silva: http://orcid.org/0000-0001-6715-474X

Andrés José Prieto: http://orcid.org/0000-0003-0604-0364

\section{References}

Brischke, C., Meyer-Veltrup, L. and Bornemann, T. (2017). Moisture performance and durability of wooden façades and decking during six years of outdoor exposure. Journal of Building Engineering, 13, 207-215. DOI: 10.1016/j.jobe.2017.08.004.

Gaspar, P. and de Brito, J. (2011). Limit states and service life of cement renders on façades. Journal of Materials in Civil Engineering, V. 23, Issue 10, pp. 1393-1404.

Prieto, A. J. and Silva, A. (2019). Service life prediction and environmental exposure conditions of timber claddings in South Chile. Building, Research and Information, 48(2), 191-206.

Sandak, A., Sandak, J., Brzezicki, M. and Kutnar, A. (2019). Portfolio of Bio-Based Façade Materials. In Biobased Building Skin (pp. 155-177). Springer, Singapore.

Silva A., de Brito J. and Gaspar P.L. (2016). Methodologies for service life prediction of buildings. With a focus on façade claddings. 1st Edition, Springer International Publishing, Switzerland. 